\title{
SIGnOS
}

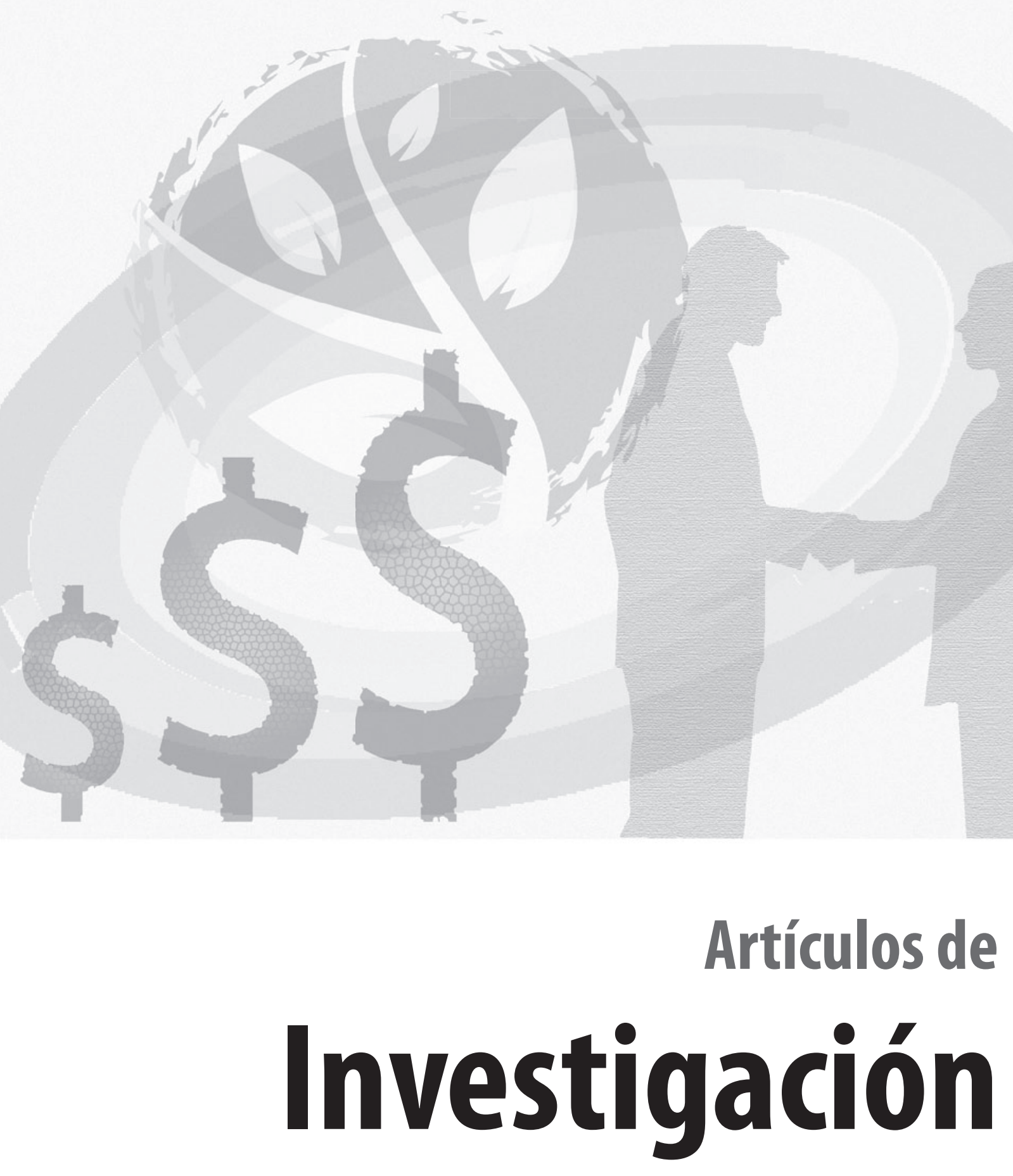




\section{Efectos de los sistemas de gestión de la calidad normalizados en las PYMES: implicaciones en la responsabilidad social empresarial ${ }^{*}$}

\section{Effects of Quality Management Systems Standard in Smes: Implications on Corporate Social Responsibility}

\begin{abstract}
Luis Humberto Barrera Tolosa**1
Yenny Zulima Vásquez Alejo

Universidad Santo Tomás e Icontec, Bogotá, Colombia

\section{RESUMEN}

El documento muestra los resultados de la aplicación de dos instrumentos (encuesta y entrevista) a la posible relación de causalidad entre el sistema de gestión de calidad y la responsabilidad social. Se busca establecer qué instrumento es más eficiente y realizar una corroboración de campo del marco teórico a manera de prueba piloto, por medio de la aplicación de la encuesta a expertos y la entrevista en profundidad en empresas. Los resultados muestran que los sistemas normalizados de gestión de la calidad y la responsabilidad en las
\end{abstract}

Recibido: 15 de junio de 2010

Revisado: 19 de agosto de 2010 Aceptado: 30 de septiembre de 2010
Artículo de Investigación.

* Correspondencia: Luis Humberto Barrera Tolosa, Ingeniero Industrial de la Universidad Libre, Especialista en Investigación Criminal, Magíster en Calidad y Gestión Integral del Convenio USTA-ICONTEC, actualmente asesor de Desarrollo Organizacional en el Equipo Líder Calidad MECI de la Policía Nacional de Colombia.

Yenny Zulima Vásquez Alejo, Odontóloga de la Universidad Javeriana. Esp. en Administración Hospitalaria, Esp. en Sistemas de Garantía de Calidad y Auditoría de Servicios de Salud, Esp. en Gestión en Salud Pública y Seguridad Social, Magíster en Calidad y Gestión Integral del Convenio USTA-ICONTEC, actualmente es responsable del Sistema de Gestión de la Calidad del Sanatorio de Agua de Dios E.S.E. pymes están mediados por los valores, los principios, las políticas, la cultura organizacional, las partes interesadas, las estrategias y las acciones, elementos que se convierten en base común, tanto para los sistemas normalizados de gestión de la calidad, como para la responsabilidad social en las empresas. Se concluye que la gestión de la calidad y la responsabilidad son el producto de esta mediación por parte de los directivos organizacionales.

Palabras clave: gestión de la calidad, grupos de interés, calidad, responsabilidad social, gestión, sostenibilidad organizacional, Sistema de Gestión de la Calidad SGC. 


\section{ABSTRACT}

The document shows the results of the application of two instruments (survey and interview) to the causal link between the quality management system and social responsibility. It seeks to establish which instrument is more efficient and perform a field corroboration of the theoretical framework as a test pilot, through the implementation of the survey to ten experts and four firms. The results show that standardized systems of quality management and accountability in SMEs are mediated by values, principles, policies, organizational culture, stakeholders, strategies and actions which are elements that become a common base for both the management system standards of quality, and for corporate social responsibility. We conclude that the quality management and accountability are the product of this mediation by organizational managers.

Key words: Quality Management, Lobbying, Quality, Social Responsibility, Management, Sustainability, SGC.

\section{INTRODUCGIÓN}

Este trabajo pertenece a una de las líneas de investigación de la Maestría en Calidad y Gestión Integral, del convenio entre la Universidad Santo Tomás (USTA) y el Instituto Colombiano de Normas Técnicas y Certificación (ICONTEC), el cual tiene como propósito identificar la relación entre el sistema de gestión de la calidad y algunos factores clave, entre ellos la responsabilidad social empresarial en las pymes colombianas.

Es reconocido que en todos los países, en especial en los que se encuentran en vía de desarrollo, las pymes contribuyen a la generación de empleo y riqueza. Como señalan Saiz ${ }^{1}$, en el caso del país Vasco, y Encina ${ }^{2}$, haciendo alusión a América Latina, sus ventas constituyen un alto porcentaje de las ventas totales, del Producto Interno Bruto (PIB) y de las exportaciones de los países. En Colombia, según Puyana ${ }^{3}$, del total de empresas existentes un $94,6 \%$ son microempresas y pymes que generan el 63\% del empleo; el Ministerio de Comercio ${ }^{4}$ señala que generan entre el $37 \%$ al $53 \%$ del PIB, y Vives ${ }^{5}$ identifica que su producción constituye el $20 \%$ de las exportaciones.

Como lo señala $\operatorname{Tar}^{6}{ }^{6}$, en diversos estudios se han descrito los éxitos y fracasos de la gestión de la calidad y se evidencia su relación con los resultados obtenidos en las pymes. Por su parte, Gómez ${ }^{7}$, Vives ${ }^{8}$

1 SAIZ, María, RODRÍGUEZ, Arturo y DE ANTONIO MATEY, Jesús. Evolución reciente de las PYMES vascas. En: Ekonomiaz: Revista Vasca de Economía. 2003, no 54, p. 128-157.

2 ENCINA HERRERA, Dickson y ARTEAGA, José. Obstáculos, logros y desafíos de las MIPYMES en Bolivia. En: Las PYMES en Latinoamérica: Estudios e investigaciones en la organización Latinoamericana de administración. Organización Latinoamericana de Administración. 2007, p. 38.

3 PUYANA, David. Las PYMES y su situación en Colombia. Avance de Investigación. Bogotá, Escuela de Negocios y Ciencias Empresariales. Universidad Sergio Arboleda, 2008, p. 1.

4 COLOMBIA. MINISTERIO DE COMERCIO, INDUSTRIA Y TURISMO. Presentación en el Seminario Iberoamericano de micro, pequeña y mediana empresa. Bogotá. Mayo 2 y 3.2005.

5 VIVES, Antonio, CORRAL, Antonio e ISUSI, Iñigo. Responsabilidad social de la empresa en las PYMES en Latinoamérica. Washington: BID, 2005, p. 14.

6 TARÍ, Juan José y MOLINA, José Francisco. Impacto de la gestión de la calidad en los resultados: un estudio empírico en empresas certificadas. Universidad de Alicante. España. 2006, p. 2.

7 GÓMEZ LÓPEZ, Javier y FERNÁNDEZ VIDAL, Lauro. Formación académica del administrador, la responsabilidad social y las PYMES. p. 223. En: Las PYMES en Latinoamérica: Estudios e investigaciones en la Organización Latinoamericana de Administración. Organización Latinoamericana de Administración. 2007. Consultado el 6 de noviembre de 2010 en http://www.eumed.net/ libros/2007b/274/indice.htm

8 VIVES, Op.cit., p. 22-70. 
y Royo ${ }^{9}$ describen los elementos que contribuyen a la sostenibilidad de las organizaciones, la satisfacción y fidelización de los clientes, grupos de interés ${ }^{10^{*}} 0$ "Stakeholders"11**, los accionistas, la comunidad, los comportamientos éticos de las empresas, entre otros, todo lo cual persigue alcanzar la generación de una buena calidad de vida laboral, con beneficio del aprendizaje individual, grupal y empresarial, y con un cumplimiento de la normatividad ambiental que persigue lograr un equilibrio con el ambiente.

En Colombia, no se cuenta con estudios de este orden en los que se mida y estandarice esta relación, por tanto, es importante contar con unos resultados, a manera de prueba piloto, que aunque no se puedan generalizar, sí favorezcan la construcción de propuestas de gestión de la calidad que den respuesta a las necesidades de las pymes colombianas y en su conjunto contribuyan al desarrollo sostenible del país.

En esta investigación, entonces, se parte de la premisa de que existe una relación de causa-efecto entre Sistema de Gestión de la Calidad (SGG) y Responsabilidad Social Empresarial (RS), y para establecer tal relación se desarrolla una revisión teórica y un trabajo de campo descriptivo sobre la realidad de las pymes. Los instrumentos utilizados son una en-

9 Royo, Teresa. (2007). La responsabilidad social empresarial. Oportunidad de mejora interna de gestión, ventaja competitiva, diferenciación y reputación de medianas y pequeñas empresas. p. 446. En: Dyna. 2007, vol. 82, no 8. p. 445-449.

10 En el presente artículo al hablar de "Grupos de interés", se debe entender integrado, los términos: "partes interesadas" o "Stakeholders", según lo aborden los diferentes autores citados, pues no se determina claramente que pretendan generar diferenciación.

11 "Al enunciar a Stakeholders, es necesario mencionar el término de Shareholders, el cual comprende a los partícipes, que serían los "públicos directamente interesados en los mecanismos de propiedad de la empresa, como son los accionistas mayoritarios y minoritarios", para así determinar su diferencia; para este grupo es importante la generación de valor, como es la rentabilidad, las ganancias, los dividendos, entre otros (Cfr. Fernández, 2005:1, Miguez, 2007:183). cuesta a expertos y una entrevista en profundidad. El propósito es identificar y analizar la relación teórica, y construir y validar instrumentos que permitan establecer la relación práctica entre SGC y el impacto sobre las expectativas de los grupos de interés, el entorno y la RS en las pymes de Colombia, con el fin de contribuir a la comprensión de las relaciones multicausales y complejas entre SGC normalizados y factores organizacionales.

\section{METODOLOGÍA}

La investigación es de tipo descriptivo, no experimental y de campo. Es importante recordar que esta investigación es la primera fase de una investigación institucional del Convenio USTA-ICONTEC. En la primera etapa se realizó una revisión al contenido de aproximadamente 504 documentos, estos elementos informativos de carácter secundario se componen de libros y revistas indexadas, trabajos de grado, entre otros, de los cuales se referencian de manera directa 62 documentos cuyo análisis se organizó en cuatro categorías: a) conceptos, b) proceso o actividades, c) resultados y d) relaciones. Este análisis se realiza con el apoyo del programa Atlas ti y lleva al planteamiento de un esquema de relaciones teóricas entre la SGC y la RS.

En la segunda etapa se desarrollan una serie de pasos que permiten formular y comprobar los instrumentos de identificación de elementos y validación del esquema de relaciones entre la SGC y la RS para las pymes colombianas. Los pasos son: identificación de variables, generación de la matriz categorial de variables (ver tabla 1), formulación de instrumentos a partir de la matriz categorial, comprobación de instrumentos, aplicación de instrumentos, organización y análisis de la información, y formulación del esquema resultante del trabajo de campo. 
Tabla 1: Matriz categorial

\begin{tabular}{|c|c|c|}
\hline FACTOR & CATEGORIAA & VARIABLE \\
\hline \multirow{9}{*}{ 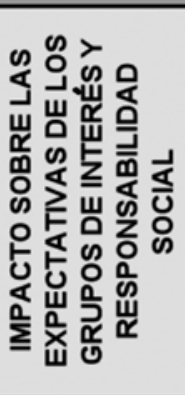 } & \multirow{2}{*}{$\begin{array}{l}\text { 1. CONCEPTUALIZACIÓN DE RS DE LA } \\
\text { EMPRESA }\end{array}$} & \begin{tabular}{|l} 
VALORES \\
\end{tabular} \\
\hline & & \begin{tabular}{|l|} 
PRINCIPIOS \\
POLLIIICAS \\
\end{tabular} \\
\hline & \multirow{3}{*}{ 2. ACTIVIDADES CONSIDERADAS RS } & CULTURA ORGANIZACIONAL \\
\hline & & ESTRATEGIA \\
\hline & & ACCIONES O INNOVACIONES \\
\hline & \multirow{3}{*}{$\begin{array}{l}\text { 3. RESULTADO O IMPACTO OBSERVADOS } \\
\text { EN RS }\end{array}$} & SOCIAL \\
\hline & & ECONÓMICO \\
\hline & & AMBIENTAL \\
\hline & 4. RELACIONES SGC y RS & SISTEMAS DE GESTIÓN: \\
\hline
\end{tabular}

Fuente: elaboración propia, 2010.

En la encuesta para la validación de contenido del esquema de relaciones se apeló a expertos para establecer si los factores del instrumento representan adecuadamente el constructo surgido de la revisión bibliográfica.

En la encuesta a expertos se tuvieron en cuenta dos aspectos: la calidad de la experticia de los encuestados y el tamaño de la muestra (cantidad de personas requeridas), lo que se definió teniendo en cuenta los trabajos de autores como Febles ${ }^{1210}$, Salas ${ }^{1311}$, Astigarraga ${ }^{14}$ y Barraza ${ }^{15}$.

12 FEBLES, Ailyn. Un modelo de referencia para la gestión de la configuración en la Pyme de Software. Tesis presentada para optar al grado de Doctor en Ciencias Técnicas. Instituto Superior Politécnico José A. Echeverri. 2003, p. 1.

13 SALAS, Mayra. Modelo Pedagógico de la Dinámica del Proceso de Formación de Gestores de Programas y Proyectos de Ciencia e Innovación. Tesis para optar al título de Doctor en Ciencias. Universidad de Oriente. Centro de Estudios Manuel F. Grant. Cuba, 2009, p. 108, 109, 130-133.

14 ASTIGARRAGA, Eneko. El método Delphi. Universidad de Deusto. Curso 2002-2003. San Sebastián. p. 10. Consultado el 6 de noviembre de 2010 en http://www.prospectiva.eu/zaharra/Metodo_delphi.pdf

15 BARRAZA, Arturo. La consulta a expertos como estrategia para la recolección de evidencia de validez basadas en el contenido. p. 12. En: Investigación Educativa Duranguense, septiembre, 2007, No. 7, p. 5-14.
Para establecer la calidad de la experticia se aplicó la siguiente fórmula:

$\mathrm{K}=(\mathrm{Kc}+\mathrm{Ka}) / 2$

Donde:

Kc: escala de 0 a 10 multiplicado por 0.1 , donde " 0 " indica no tener ningún conocimiento.

$K a$ : Evaluación del experto en: alto, medio o bajo.

$\mathrm{K}$ : competencia, la cual se clasifica de acuerdo con los siguientes criterios:

$0,8<\mathrm{K}<1,0 \quad$ Coeficiente de competencia alto

$0,5<\mathrm{K}<0,8 \quad$ Coeficiente de competencia medio

$\mathrm{K}<0,5 \quad$ Coeficiente de competencia bajo

Con base en este procedimiento se seleccionan los expertos de competencia alta, sin embargo, cuando no existe suficiente número de expertos con esta condición se pueden tomar los encuestados cuyo promedio es igual o mayor a 0,8 ; razón por la cual en este trabajo se tomaron seis expertos de competencia alta y cuatro de competencia media.

Para determinar el tamaño de la muestra de expertos se tiene en cuenta el método Delphi. Según éste, se 
considera que el número de expertos mínimo es de 7 y el máximo de 30. La fórmula utilizada fue la siguiente:

$\mathrm{n}=\quad\left[\mathrm{N}\left(\mathrm{i}^{2} \mid k\right)+\mathrm{N}\left(\mathrm{p}-\mathrm{p}^{2}\right)\right]$

$$
\mathrm{N}\left(\mathrm{i}^{2} \mid k\right)+\mathrm{p}-\mathrm{p}^{2}
$$

Donde:

i: nivel de precisión

N: población estimada (de expertos)

n: muestra (cantidad de personas)

p: proporción del error

k: constante fijada a partir del nivel de confianza

Con dicha información se determinó el número preliminar de expertos (n) para un nivel de confianza del 99\%. Como muestra para la encuesta se estableció un número de diez expertos a quienes se les aplicaron los instrumentos. La comunicación con ellos fue por vía telefónica, electrónica o presencial, se les entregó el cuestionario de autovaloración de expertos y el de comprobación del esquema de relación.

Para la selección de la muestra piloto de las empresas, en la entrevista se tuvo en cuenta lo siguiente:

Estar certificadas o tener implementados un SGC bajo la norma ISO 9001:2008 y ser pymes.

El número PYMES para la prueba piloto se determinó de acuerdo con los recursos de los investigadores. Se definió que serían cuatro.

En cada empresa se seleccionaron tres partes interesadas: gerente, empleados y cliente.

Se llevó a cabo previamente una sensibilización que definiera la posibilidad de participación en la investigación.

Grabar las entrevistas.
A los instrumentos se les realizó un análisis de confiabilidad por medio del método Alpha de Cronbach, el cual dió 0,7748 en la encuesta a expertos y para el análisis de la matriz de convergencias, divergencias y enunciados tipo para la entrevista a profundidad, lo cual mostró que los instrumentos son confiables para las variables a analizar en la relación entre SGC y RS.

Con los datos obtenidos referentes a la validación del constructo ${ }^{16^{*}}$, producto de la encuesta, se realizó un primer ejercicio manual en hoja de cálculo Excel, para obtener los promedios de las respuestas de cada experto en cada una de las categorías establecidas y, posteriormente, se parametrizaron los datos en el Paquete Estadístico de Propósitos Múltiples (SPSS), en el que se aplicó el coeficiente de correlación Rho de Spearman, para determinar el grado de asociación entre variables.

En el caso de la entrevista a profundidad las grabaciones se transcribieron en la matriz, que comprende: las preguntas, las respuestas de los entrevistados, las columnas para registrar las convergencias, las divergencias y, por último, los enunciados tipo como resultado de las dos anteriores.

\section{RESULTADOS Y DISCUSIÓN}

\section{Encuesta a expertos y esquema de relaciones resultante}

Debido a la gran cantidad de relaciones que se pueden dar del análisis de los resultados del Rho de Spearman, sólo se enuncian las correlaciones positivas fuertes.

En la primera categoría se encuentran relaciones positivas muy fuertes entre la adopción e implementación de

16 Entiéndase constructo como la representación o esquema teórico de la relación de causalidad del SGC y la RS. 
las políticas y la identificación del enfoque de la organización en materia de RS. Igualmente, existe relación positiva muy fuerte entre la identificación del enfoque y el uso de las políticas organizacionales para la resolución de los conflictos con el cliente y las partes interesadas.

Respecto a las acciones, los expertos relacionan la promoción del desarrollo del talento humano en la organización con la RS y valoran los mecanismos de participación hacia el cliente y las partes interesadas con los que cuenta la organización.

Los resultados o beneficios que generan las actividades de RS se correlacionan muy fuerte con el mejoramiento del clima organizacional, los beneficios económicos esperados por la alta dirección, el mejoramiento de los procesos, la calidad de los bienes y servicios, las relaciones de confianza y fidelidad con los clientes y los proveedores, así mismo con el favorecimiento del equilibrio entre la vida laboral y familiar de los colaboradores.
Llama la atención la poca correlación que hay entre la $\mathrm{RS}$ y la generación de ventajas competitivas, pues esto es controvertido en el marco referencial, especialmente, cuando el tema es tratado por expertos europeos e incluso norteamericanos.

En la categoría de relaciones entre los SGC y la RS es en la que hay mayor correlación de las variables; la correlación es media cuando se pregunta por el impacto en la sostenibilidad de la empresa; al incorporar elementos de $\mathrm{RS}$ a la estrategia se torna positiva, y respecto a que los sistemas normalizados de calidad impactan a la RS en las organizaciones, la correlación es muy fuerte. Así mismo, se observa una correlación media cuando se analiza la contribución de los sistemas de gestión en la RS en las organizaciones, esto puede ser debido a que ellas van más allá y ofrecen mayor cobertura y ámbito de aplicación. Existen, pues, elementos comunes entre los SGC y la RS, tal como se observa en la figura 1 , lo cual concuerda con los resultados de la revisión bibliográfica inicial.

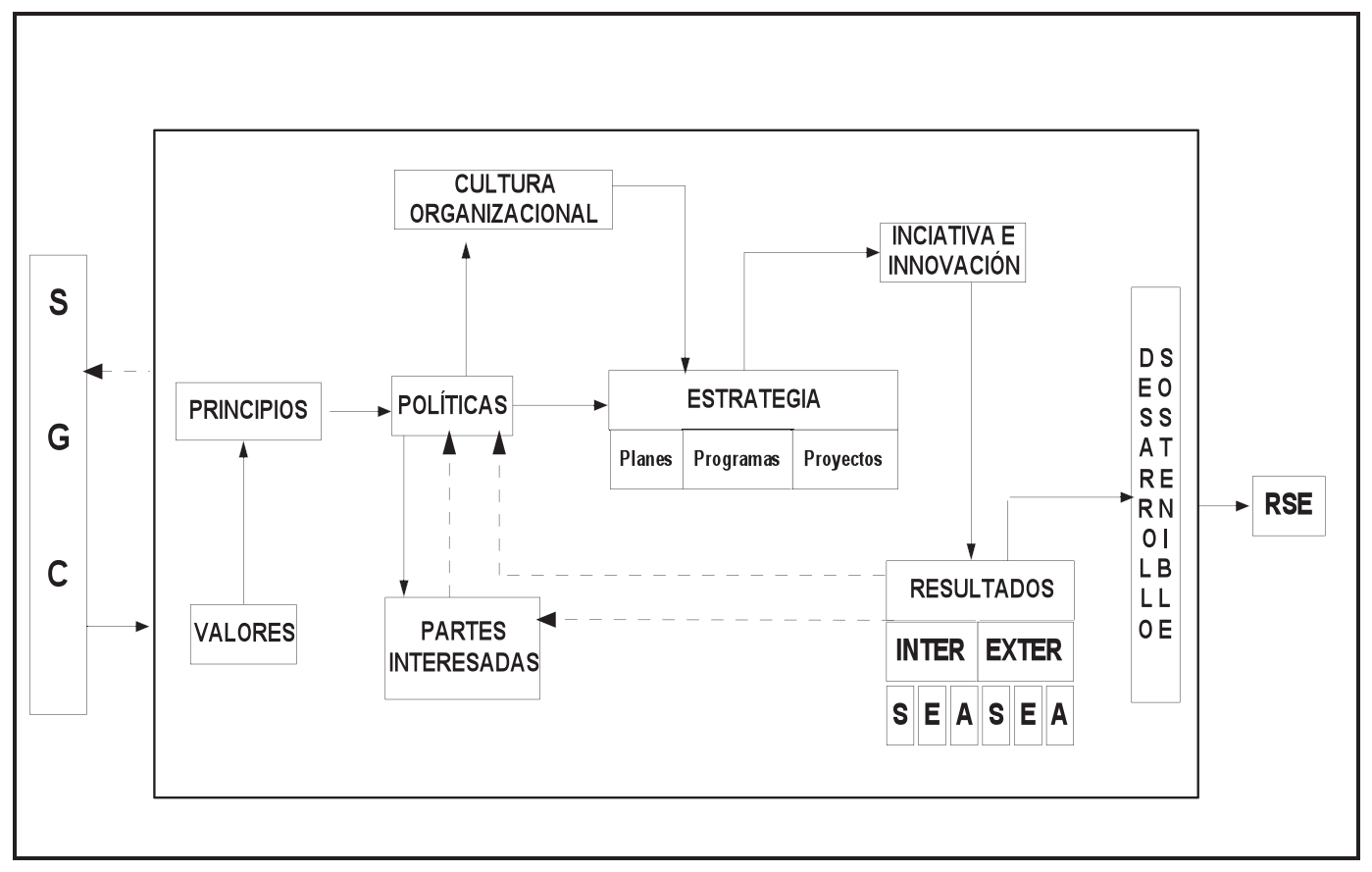

Figura 1. Relación de causalidad SGC y RS resultante de la encuesta - Fuente: Elaboración propia, 2010. 


\section{Entrevista y esquema de relaciones resultante}

En los resultados de las entrevistas efectuadas a las tres partes interesadas: gerentes, empleados y clientes se observa que la mayoría de las empresas tienen valores, principios y políticas formalmente adoptadas, sin embargo, aunque la mayoría de los encuestados conocen las políticas, sólo algunos dan cuenta de los valores y principios. De la misma manera, los resultados muestran que los diferentes grupos de interés no cuentan con conocimiento similar de las organizaciones en las que se encuentran.

$\mathrm{Al}$ revisar las actividades consideradas como de RS por parte de los encuestados, se encuentra que hacen mención de actividades como el cumplimiento legal, las actividades ambientales, la educación social, la estabilidad e incentivos laborales, la participación de las partes y el cumplimiento del objeto social.

$\mathrm{Al}$ analizar el grupo de los gerentes, la totalidad encuentra actividades relacionadas con RS. La mayoría corresponde a temas ambientales, algunos hacen alusión a lo referente con ley y unos pocos a las donaciones, cumplimiento del objeto social, incentivos laborales, bienestar del trabajador y estabilidad laboral.

El grupo de empleados también encuentra actividades de RS. Todos estiman el cumplimiento de la ley y los temas ambientales, el bienestar del trabajador y el bienestar social, y algunos la ven en el hecho de dar donaciones, la calidad del servicio y la participación en la planificación de la empresa. Por último, en el grupo de los clientes hallan actividades de RS que corresponden al cumplimiento de la ley y la calidad del servicio.

Como resultado, de lo anterior se puede afirmar que las partes consultadas mencionan actividades de responsabilidad que se pueden encasillar en lo "mínimo" de lo que corresponde a RS, como es el caso del cumplimiento de la ley. Esto significa que las partes perciben como un gran logro entregar lo mínimo, y dejan la sensación de que en ocasiones no se cumpla ni siquiera con lo establecido por ley.

$\mathrm{Al}$ analizar los beneficios que trae a las organizaciones realizar acciones de RS, se obtiene que la mayoría de los encuestados identifican beneficios para la empresa, como el posicionamiento o participación en el mercado, la buena imagen, la fidelidad del cliente y de los trabajadores, el mejoramiento de la cultura organizacional, la rentabilidad, la mejora de procesos.

Con base en lo anterior, se puede afirmar que las empresas pueden ver como una buena inversión las actividades de RS en razón a que les retribuye en una diversidad de formas que permiten la sostenibilidad de la organización: buena imagen, funcionamiento en el mercado, fidelización de los empleados y clientes, mejoramiento de la cultura y clima organizacional, ventajas competitivas, aumento de la rentabilidad y la productividad

$\mathrm{Al}$ analizar los impactos obtenidos, se observa que la mayoría hallan impactos económicos. Como la generación de empleo, la disminución de los costos, la participación en el mercado, la sostenibilidad de la empresa, la generación de impuestos y el mejoramiento de los productos por innovación.

Se encuentran impactos sociales positivos como: suplir necesidades básicas esenciales, incremento del bienestar de la comunidad, desarrollo de los trabajadores, disminución del consumo de agua, manejo de vertimientos y disminución de la carga biológica, química y de residuos sólidos. Sin embargo, también se mencionan impactos negativos como: aumento del ruido, aumento de material particulado y daño al paisaje. 
$\mathrm{Al}$ analizar lo señalado por el grupo de los gerentes, la totalidad de ellos hallan los tres tipos de impactos: sociales, económicos y ambientales. Entre los económicos encuentran la participación del mercado, la generación de empleo, la disminución de precios al usuario y el pago de impuestos. En lo ambiental señalan el aumento del ruido, de material particulado y daño al paisaje, En lo social identifican: el suplir necesidades básicas esenciales, el incremento del bienestar de la comunidad y el empleo.

En el grupo de los empleados la mayoría encuentra también los tres grupos de impacto. Entre los económicos, la generación de empleo; en lo ambiental, la disminución de las cargas (química, biológica, residuos sólidos); y en lo social, el bienestar general.

En el grupo de los clientes la totalidad halla sólo dos tipos de impacto: económico, como la generación de empleo, y el ambiental, descrito como la reducción de los riesgos de los residuos. Es de anotar que no hallan ningún impacto de orden social.

Como resultado de lo anterior se puede afirmar que las empresas, sean de bienes o servicios, reportan impactos sociales, económicos y ambientales, y se percibe en mayor grado el económico, siendo la generación de empleo el que presenta un lugar preponderante, ya que ocupa más de la mitad de los impactos. El segundo lugar corresponde al manejo de residuos biológicos y químicos, y en tercer lugar está el impacto de orden social.

Con referencia a los SGC que utilizan las organizaciones se encuentra que la gran mayoría de los encuestados afirma que se realiza bajo los parámetros de la norma ISO 9001:2008.

A la pregunta de cuál es la articulación entre SGC y la $\mathrm{RS}$, se encuentra que algunos dicen que la gestión de la calidad conduce a la responsabilidad social, otros consideran que corresponde al cumplimiento con los clientes, unos pocos encuentran que el SGC es un medio para alcanzar la RS, que la norma de calidad debe contemplar los elementos clave de RS o que la norma de calidad y la RS van de la mano, ya que ambas propenden por el mejoramiento del entorno.

Lo anterior permite afirmar que la mayoría considera que existe una articulación entre SGC y RS, como lo señala la totalidad de los gerentes y empleados y la mayoría de los clientes. Sin embargo, se difiere en el modo de articulación. Por tanto, se puede proponer que dependiendo de la manera o proceso como se planifique e implemente el SGG de las empresas, éste puede conducir a expresiones o resultados que se enmarquen como de RS.

Como resultado de la entrevista, se observa que existen los mismos elementos planteados teóricamente, pero no operan de manera similar, la operación que se aprecia se muestra en la figura 2. En ella se puede notar que los valores se mencionan, mas no se establece de manera clara qué direccionan, y los principios parecen no subordinarse a los valores, sino que presentan una mutua relación con las políticas. En términos generales, los demás elementos mantienen el marco teórico propuesto.

Al analizar el enfoque de RS en las organizaciones encuestadas predomina el clásico, lo que confirma lo expuesto por Cabrera ${ }^{17}$ acerca de una visión primaria de la responsabilidad social. Quiere esto decir que las organizaciones se enfocan con el objetivo de la maximización del beneficio y consideran que la implicación social genera un costo sin beneficio real, es decir, se enfrenta un enfoque que no asume responsabilidad

17 CABRERA, María Katiuska, DÉNIZ, María de la Cruz y SANTANA, Domingo Javier. Responsabilidad social corporativa y empresa familiar. En: Revista Europea de Dirección y Economía de la Empresa. 2005, vol.14, no 4, p. 43- 58. 


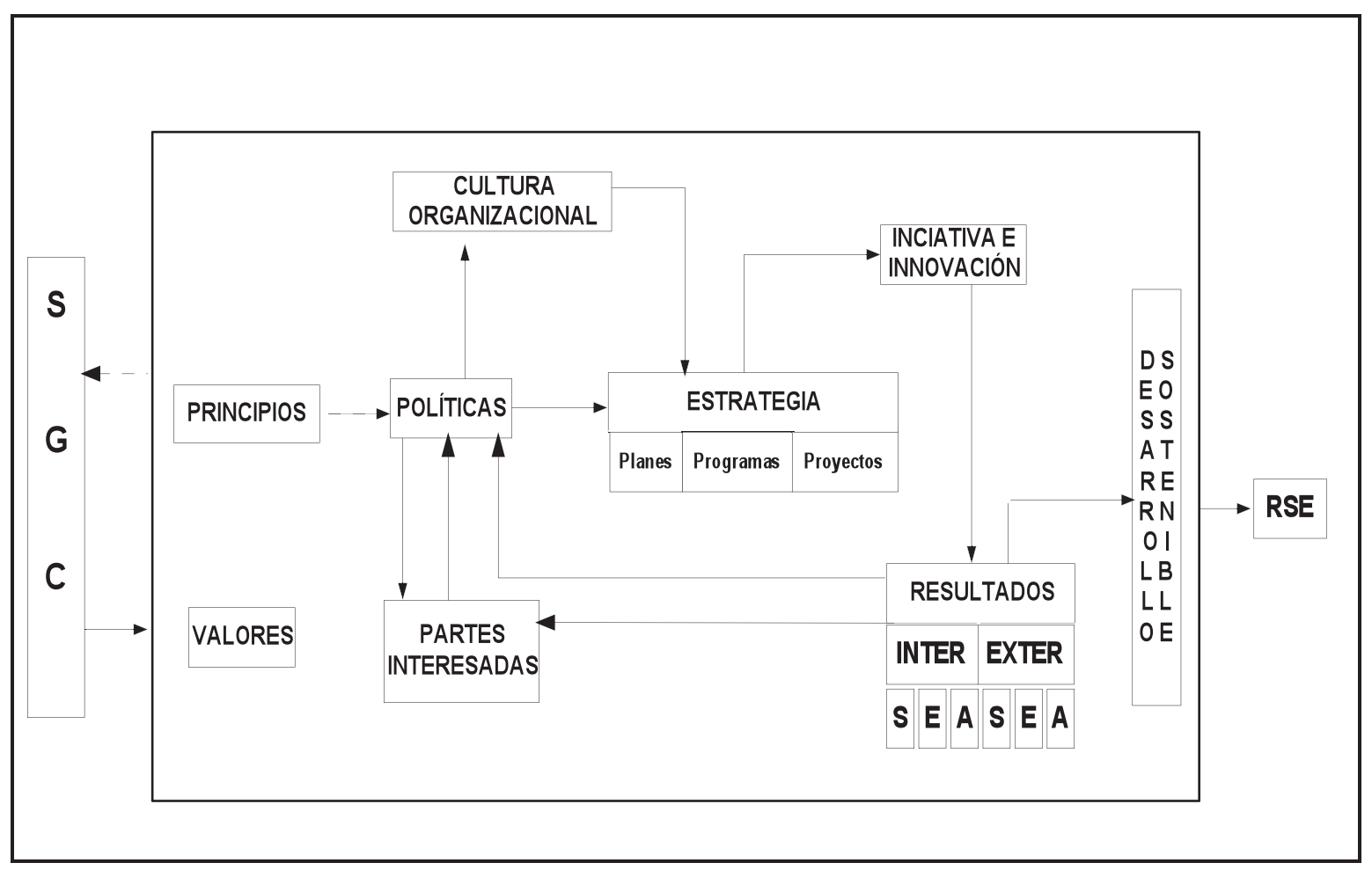

Figura 2. Relación de causalidad SGC y RS resultante de la entrevista - Fuente: Elaboración propia,, 2010.

en el ámbito social. La relación fuerte en este aspecto confirma lo estimado por Friedman en su postulado instrumental, según el cual las organizaciones aún desconocen la función real de ellas con el componente de sostenibilidad y se deben concentrar en las utilidades. Este concepto es divergente en lo que respecta al reconocimiento de que las inversiones sociales contribuyen con la riqueza social y el bien común.

Al indagar sobre el nivel de comprensiones empresariales sobre RS es evidente la ausencia de consenso en torno a una definición conceptual como lo confirma lo referido por el $\mathrm{CCRE}^{18}$, quiere esto decir que aunque existe un nivel de acuerdo significativo en torno a los elementos que esta responsabilidad debería comprender, las organizaciones continúan relacionándola con

18 COLOMBIA. CENTRO COLOMBIANO DE RESPONSABILIDAD EMPRESARIAL (CCRE). Estudio Línea de Base sobre Responsabilidad Social Empresarial en Colombia. Bogotá, 2006. los compromisos hacia los empleados y la comunidad, en primer plano. Se observa también que la RS en las organizaciones encuestadas presenta divergencias en lo referente al compromiso con los clientes, el ambiente y la sostenibilidad, y se dejan de lado las políticas del gobierno y la ética que de ello se deriva.

\section{CONCLUSIONES}

La presente investigación fue correlacional, puesto que evaluó el grado de relación de dos categorías y sus variables: la gestión de la calidad a través de la implementación de un sistema normalizado y la responsabilidad social empresarial.

La utilidad de la prueba de coeficiente de correlación de Spearman en el campo de los sistemas de gestión consistió en aportar una respuesta cuantificable a la relación entre las variables bajo estudio. 
Sin importar si estos elementos son modificados o complementados como producto de la integración de los resultados de los diferentes factores del proyecto institucional, su utilización en la siguiente fase del proyecto es pertinente y adecuada para la validación del esquema de relaciones resultante.

Si bien la implementación y certificación de sistemas normalizados de gestión de la calidad mejora la imagen de calidad a las empresas, el beneficio inmediato que se evidencia es el mantenimiento de su participación en el mercado. Pero si las organizaciones desarrollan actividades de RS, los beneficios se amplían al mejoramiento de la confianza con el cliente y con los demás grupos de interés, lo que genera la fidelidad y solidaridad de ellos en el ámbito interno y externo, lo cual puede abrir la posibilidad de ingresar a nuevos mercados, mejorar la capacidad de la empresa para entregar sus productos o prestar servicios y, además, generar la satisfacción de expectativas como valor distintivo y decisorio que marca la diferencia en el mercado.

Entre las razones por las cuales las pymes desarrollan actividades de RS está el buscar la armonización de los beneficios privados relacionados con los intereses del dueño o los accionistas, y los beneficios sociales, especialmente, en lo relacionado con sus clientes internos a quienes la empresa busca mejorarles la calidad de vida.

Si bien la RS como término es nuevo, más no como práctica, en la actualidad tiene un auge inusitado relacionado con la magnitud de los problemas sociales y ambientales que impactan negativamente a los mercados.

Existen teóricamente unos elementos que presentan las relaciones entre SGC y la RS.

Los elementos y sus relaciones son concebidos de esta manera: los fundamentos (filosofías y creencias) generan los valores, de éstos se toman los que se convierten en principios, éstos son rectores de las políticas que actúan sobre las partes interesadas y la cultura organizacional, influyendo en las estrategias de acción y, a través de ellos, las iniciativas e innovaciones que se reflejan en los resultados sociales, ambientales y económicos y que generan la sostenibilidad.

El resultado de la encuesta a los expertos muestra que los elementos de relación propuestos en el esquema teórico son considerados por ellos de la misma forma, tanto en sus componentes como en las realcuaines.

También, es posible ver que las concepciones teóricas son la base para proponer instrumentos que midan y contribuyan a determinar, cuáles y de qué manera esos elementos teóricos son tenidos en cuenta por las pymes para la gestión de la calidad y cómo incide ésta en la RS de ellas. Con esto se puede contrastar entre lo teórico y lo aplicado en las pymes como se observa en la figura 1, donde se muestra que los elementos propuestos en el esquema teórico se mantienen, sin embargo, las relaciones presentan variaciones en la categorización y secuencialidad.

Es notorio que los valores que provienen de los fundamentos filosóficos y las creencias, y los cuales se consideran base fundamental en esta propuesta, no se perciben en las pymes de esta manera; en cambio, sí existe claridad con respecto a las políticas como rector del actuar.

La aplicación de los instrumentos muestra que es posible encontrar a través de ellos los elementos que median la relación entre SGC y RS en las pymes, además se puede observar la jerarquización u orden concebido por éstas.

Todas las pymes incluidas en este estudio generan planes, programas o proyectos que conducen a acciones con los respectivos resultados, y se tiene conocimiento acerca de los beneficios o perjuicios. Se observa tam- 
bién que mantienen una mirada muy clásica de concebir la RS y, por ende, la gestión de calidad implementada entregará como resultados una visión reducida de ella, porque sólo la tiene en cuenta si le genera unos beneficios económicos, debido a que considera que la empresa no tiene este tipo de responsabilidad en el ejercicio de la operación.

\section{RECOMENDACIONES}

Se deberia continuar con la segunda fase la investigación, para aplicar de forma amplia y representativa los instrumentos y metodologías propuestas en este trabajo, de modo que se genere un conocimiento que facilite la implementación de la $\mathrm{RS}$ en la pymes colombianas.

Teniendo en cuenta que la mayoría considera el cumplimiento de la Ley como el punto de partida de la RS y, además, el acatamiento de las normas es la base de dicha responsabilidad, el Estado debería propender por ese sentido con el propósito de generar con mayor celeridad la $\mathrm{RS}$ de las pymes de Colombia.

La academia debería debatir y ser foro impulsor de la RS como un deber ético de las empresas y de las pymes, en particular.

En los currículos de formación básica, vocacional y superior este tema debería ser incorporado como parte fundamental del saber, saber hacer y saber ser del educando.

\section{BIBLIOGRAFÍA}

ASTIGARRAGA, Eneko. El método Delphi. Universidad de Deusto. Curso 2002-2003. San Sebastián. Consultado el 6 de noviembre de 2010 en http:// www.prospectiva.eu/zaharra/Metodo_delphi.pdf
BARRAZA, Arturo. La consulta a expertos como estrategia para la recolección de evidencia de validez basadas en el contenido. En: Investigación Educativa Duranguense, septiembre, 2007, no. 7, p. $5-14$.

CABRERA, María Katiuska, DÉNIZ, María de la Cruz y SANTANA, Domingo Javier. Responsabilidad social corporativa y empresa familiar. En: Revista Europea de Dirección y Economía de la Empresa. 2005, vol.14, no 4, p. 43- 58.

COLOMBIA. CENTRO COLOMBIANO DE RESPONSABILIDAD EMPRESARIAL (CCRE). Estudio Línea de Base sobre Responsabilidad Social Empresarial en Colombia. Bogotá, 2006.

COLOMBIA. MINISTERIO DE COMERCIO, INDUSTRIA Y TURISMO. Presentación en el Seminario Iberoamericano de Micro, Pequeña y Mediana Empresa. Bogotá. Mayo 2 y 3. 2005.

ENCINA HERRERA, Dickson y ARTEAGA, José. Obstáculos, logros y desafíos de las mipymes en Bolivia. En: Las pymes en Latinoamérica: Estudios e investigaciones en la organización Latinoamericana de administración. Organización Latinoamericana de Administración. 2007.

FEBLES, Ailyn. Un modelo de referencia para la gestión de la configuración en la pyme de Software. Tesis presentada para optar al grado de Doctor en Ciencias Técnicas. Instituto Superior Politécnico José A. Echeverri. 2003.

FERNÁNDEZ, Pablo. Valoración de empresas: cómo medir y gestionar la creación de valor. $3^{\mathrm{a}}$. ed. Barcelona: Gestión, 2000, 2005, p. 931.

GÓMEZ LÓPEZ, Javier y FERNÁNDEZ VIDAL, Lauro. Formación académica del administrador, la responsabilidad social y las pymes. En: Las pymes 
en Latinoamérica: Estudios e investigaciones en la Organización Latinoamericana de Administración. Organización Latinoamericana de Administración. 2007. Consultado el 6 de noviembre de 2010 en http://www.eumed.net/libros/2007b/274/ indice.htm

MIGUEZ GONZÁLEZ, María Isabel. Análisis del uso de los conceptos de público, stakeholder y constituent en el marco teórico de las relaciones públicas. En: ZER, noviembre, 2007, vol. 12, no 23, p. 183-197.

PEÑA, Guillermo y MARTÍNEZ, Gerardo. Integración de la responsabilidad social con el modelo ISO 9001 en pymes colombianas certificadas. En: SIGNOS, junio, 2009, vol. 1, no 1, p. 11-32.

PUYANA, David. La pyme y su situación en Colombia. Avance de Investigación. Bogotá, Escuela de Negocios y Ciencias Empresariales. Universidad Sergio Arboleda, 2008, p. 31.

ROYO, Teresa. (2007). La responsabilidad social empresarial. Oportunidad de mejora interna de gestión, ventaja competitiva, diferenciación y re- putación de medianas y pequeñas empresas. En: Dyna. 2007, vol 82, no 8, p. 445-449.

SAIZ, María, RODRÍGUEZ, Arturo y MATEY DE ANTONIO, Jesús. Evolución reciente de las pymes vascas. En: Ekonomiaz: Revista Vasca de Economía. 2003, no 54, p. 128-157.

SALAS, Mayra. Modelo Pedagógico de la Dinámica del Proceso de Formación de Gestores de Programas y Proyectos de Ciencia e Innovación. Tesis para optar al título de Doctor en Ciencias. Universidad de Oriente. Centro de Estudios Manuel F. Grant. Cuba, 2009, p. 174.

TARÍ, Juan José y MOLINA, José Francisco. Impacto de la gestión de la calidad en los resultados: un estudio empírico en empresas certificadas. Universidad de Alicante. España, 2006.

VIVES, Antonio, CORRAL, Antonio e ISUSI, Iñigo. Responsabilidad social de la empresa en las pymes en Latinoamérica. Washington: BID, 2005, p. 186. 